$\begin{array}{ll}\text { Research Square } & \begin{array}{l}\text { Preprints are preliminary reports that have not undergone peer review. } \\ \text { They should not be considered conclusive, used to inform clinical practice, } \\ \text { or referenced by the media as validated information. }\end{array}\end{array}$

\title{
Clinical and Economic Consequences of Switching From Omalizumab to Mepolizumab in Uncontrolled Severe Eosinophilic Asthma: an Observational Retrospective Study
}

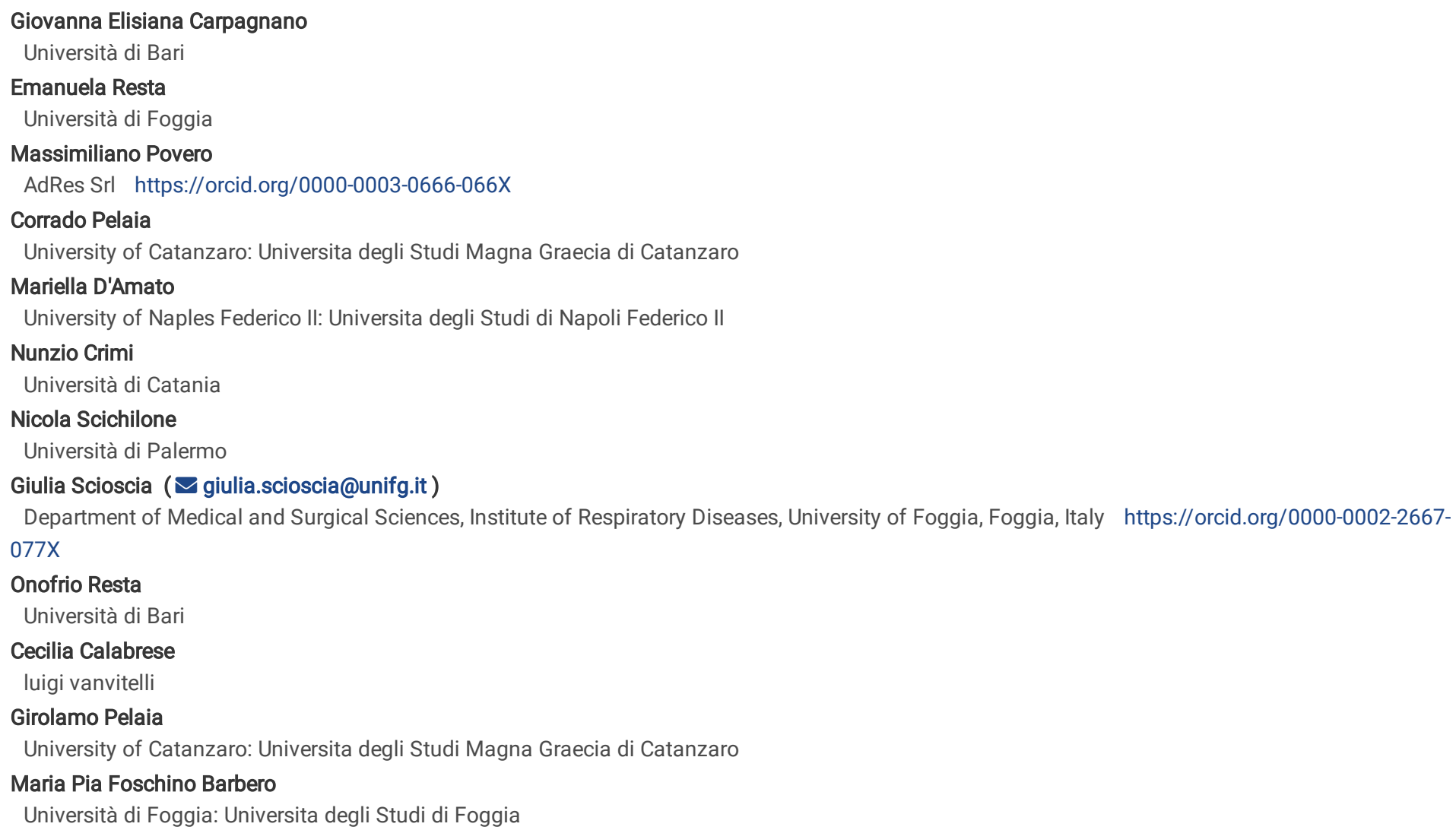




\section{Abstract}

Background: Severe asthma is burdened by frequent exacerbations and use of oral corticosteroids, which worsen patients' health and increase healthcare spending. The aim of this study was to assess the clinical and economic impact of switching from omalizumab to mepolizumab in patients eligible for both biologics, but not optimally controlled by omalizumab.

Methods: We retrospectively enrolled uncontrolled severe asthmatic patients, referred to seven asthma clinics in Italy, who switched from omalizumab to mepolizumab during the last two years. Clinical, functional, and laboratory information included blood eosinophil count, asthma control test, spirometry, serum IgE, fractional exhaled nitric oxide, oral corticosteroids intake, use of controller and rescue drugs, exacerbations/hospitalizations, visits and diagnostic exams. Within the perspective of Italian National Health System, a pre- and post-mepolizumab 12-month standardized total cost per patient was calculated.

Results: 33 patients were enrolled: 5 males and 28 females, mean age 57 years, mean disease onset 24 years. At omalizumab discontinuation, $88 \%$ were oral corticosteroids-dependent with annual mean rate of 4.0 clinically significant exacerbations, 0.30 exacerbations needing emergency room visits or hospitalization; absenteeism due to disease was 10.4 days per patient. Switch to mepolizumab improved all clinical outcomes, reducing total exacerbation rate $(\mathrm{RR}=0.06,95 \% \mathrm{Cl} 0.03$ to 0.14$)$, oral corticosteroids -dependent patients $(\mathrm{OR}=0.02,95 \% \mathrm{Cl} 0.005$ to 0.08$)$, and the number of lost working days because of uncontrolled disease $(\Delta=-7.9,95 \% \mathrm{Cl}-11.2$ to -4.6$)$. Pulmonary function improved, as well as serum IgE, fractional exhaled nitric oxide and eosinophils decreased. Mean annual costs were $€ 12,239$ for omalizumab and $€ 12,639$ for mepolizumab $(\Delta=€ 400,95 \% \mathrm{Cl}-1,588$ to 2,389$)$; the increment due to drug therapy $(+€ 1,581)$ was almost offset by savings regarding all other cost items $(-€ 1,181)$.

Conclusions: Patients with severe eosinophilic asthma, not controlled by omalizumab, experienced comprehensive benefits in asthma control by switching to mepolizumab. These relevant improvements were burdened by only very slight increases in economic costs.

\section{Background}

Severe asthma in the era of personalized medicine can benefit from the newest therapeutic approaches that are changing the natural disease course. However, severe asthma still accounts for approximately half of asthma-associated healthcare costs [1]. The most important costs of severe asthma are mainly due to management of not controlled patients in terms of exacerbations, frequent access to health services, drug consumption, side effects of oral corticosteroids (OCS) use, treatment of comorbidities and losses from missed work and school days [2, 3]. Most of these clinical aspects further extend beyond direct medical costs, and are also associated with personal problems leading to significant worsening of health-related quality of life (HRQoL), which negatively impacts on patients and caregivers [4]. Furthermore, an estimated one third of asthma-related deaths occur in patients previously hospitalized for exacerbations, thereby indicating a high mortality risk in severe uncontrolled asthma [3].

GINA (Global Initiative for Asthma) guidelines recommend at step 5 the use of biologics before OCS, when maximum dosages of dual therapy based on inhaled corticosteroids (ICS) and long-acting $\beta_{2}$-adrenergic agonists (LABA), eventually integrated by other controller drugs, do not allow to obtain an adequate disease control [2]. However, although more than half of severe asthmatic patients are eligible to at least one of available biologics, most of these subjects are still not using such therapeutic agents, thus remaining uncontrolled. With regard to both patient and physician concerns due to a poor knowledge about biologics, the cost of these new drugs discourages payer policies and often represents the main cause of their limited utilization in clinical practice [4]. However, real-life studies demonstrated that the pheno-endotype guided use of biologics is globally cost-effective in responders, thereby reducing overall costs of severe asthma management.

For more than a decade, the only available biologic therapy for severe uncontrolled allergic asthmatics was omalizumab (OMA), sometimes prescribed also in patients who did not represent the ideal targets as their asthma was only partially driven by allergy [5]. Although OMA resulted to be effective in lowering clinical exacerbations and symptoms, thus improving lung function, sparing OCS and bettering quality of life (QoL) [6], therapeutic effectiveness was only partial in some patients, so that the cost of this drug should be added to the burden of standard management of uncontrolled asthma. The cost of an add-on biological treatment cannot be completely justified when it is not fully effective. This can happen when our therapeutic choices are not perfectly appropriate, with the consequence that a given biologic does not provide an adequate asthma control and further increases healthcare expenditures.

The introduction of new biologic therapies with different mechanisms of action and targets, such as mepolizumab (MEP), recently changed the therapeutic scenario of severe asthma and is making it possible for clinicians to operate a reasoned choice [6-8]. It is today imperative to select the biologic that could provide the best possible response by targeting the recognized biological pathway underlying the specific patient phenotype [9]. Availability of several biologics also allows an eventual switch from one drug to another in unresponsive patients, with probable clinical and economic advantages. Several studies analyzed the cost-effectiveness of OMA and MEP, but contrasting results about this relevant topic have been obtained [3, 5]. Although experiences on switches from a biologic to another, motivated by poor efficacy of the first prescribed drug, are now common and several authors have described the clinical benefits provided by changing OMA with MEP in selected severe asthmatic patients [10, 11], to our knowledge no studies have been published about the relative economic impact.

The aim of the present study was therefore to explore, for the first time, the economic consequences detectable in subjects with severe eosinophilic asthma, undergoing a switch from OMA to MEPO because eligible for both these biologics, but not optimally controlled by OMA. In order to pursuing this 
objective, we analyzed important clinical aspects driving asthma-related expenses referring to a 12-month standardized pre- and post- MEP total cost, within the perspective of Italian National Health System (NHS).

\section{Materials And Methods}

\section{Patients}

All patients > 12 years old with uncontrolled severe eosinophilic asthma, who referred to 7 asthma university clinics in Italy and switched from OMA to MEP during the last two years, were retrospectively enrolled in the present study. The relevant clinical parameters were collected at three time points: before starting OMA (pre-OMA), before starting MEP (pre-MEP), and after about one year of MEP (post-MEP). Collected information included blood eosinophil count, asthma control test (ACT) score, pulmonary function, serum IgE levels, fractional exhaled nitric oxide (FeNO), OCS intake, consumption of controller and rescue drugs, number of exacerbations and hospitalizations, unscheduled visits and diagnostic exams, and number of lost working days because of the disease. All patients started OMA due to severe uncontrolled allergic asthma, and switched to MEP if considered to be non-responders because of one or more of the following reasons: i) they could not discontinue, or even needed to escalate, the daily dose of OCS; ii) they experienced $>2$ exacerbations or > 1 hospitalization per year. Switch to MEP was performed according to 2019 GINA guidelines [12].

Ethical Committee approval was not needed because the drugs were prescribed according to current medical practice as part of the standard of care. All patients signed a written informed consent for the treatment of personal data, which were anonymized.

\section{Economic evaluation}

The annualized total cost of resource consumption, exacerbation management, pharmacologic therapies, and productivity loss were investigated during treatment periods with either OMA or MEP, in order to estimate the economic impact due to switching to MEP.

Cost of MEP treatment was evaluated considering 1 subcutaneous $100 \mathrm{mg}$ vial every 4 weeks [13], while the appropriate doses and administration frequencies of OMA were determined according to body weight and baseline serum IgE levels, measured before starting treatment. [14]. The cost of both therapies for each treatment cycle was calculated according to the ex-factory price [15], applying the mandatory discount ( $5 \%+5 \%$ reduction) and price reductions negotiated among the pharmaceutical company, Local Health Units, and Hospital Units [16]. The costs of ICS/LABA, OCS, long-acting muscarinic antagonist (LAMA), short-acting $\beta_{2}$-adrenergic agonists (SABA), and leukotriene inhibitors were calculated using the reference prices established by negotiation for each product package [17], as well as for the specific posology referring to each patient (Table S1 in Additional file 1). The cost of OCS therapy was calculated considering only prednisone, as the use of OCS was expressed in prednisone dose equivalents, assuming the best package fitting the daily dosage.

The cost of exacerbations treated only with drug therapy was calculated considering, if specified, the expense for one cycle of antibiotic therapy; no OcS extra therapy was considered since all patients were already treated with OCS. The tariff of $€ 280$ for an emergency room visit was applied for severe exacerbations not needing hospitalization [18], while for hospitalized exacerbations we considered the specific tariff of Diagnosis Related Group (DRG) reported in patient medical record [19], detailed in hospital admission longer than 1 day $(\mathrm{H})$ and day-hospital (DH). Specifically, DRG 96 "Bronchitis and asthma, age > 17 years with complications" ( $€ 2,537$ for $\mathrm{H}$ and $€ 198$ for DH), DRG 97 "Bronchitis and asthma, age $>17$ years without complications" ( $€$ 1,832 for $\mathrm{H}$ and $€ 197$ for DH), and DRG 98 "Bronchitis and asthma, age < 18 years" ( $€ 1,538$ for $\mathrm{H}$ and $€ 185$ for DH) were considered, respectively. Costs for specialist visits ( $€$ 12.91, cod 89.01) and diagnostic exams were evaluated using National tariffs [19]. Tests and exams included spirometry ( $€$ 37.18, cod 89.37.2), post bronchodilator test ( $€$ 37.18, cod 89.37.4), serum IgE level measurement ( $€ 71.18$, cod 90.68.1), FeNO detection (assumed same cost of spirometry), blood eosinophil number ( $€ 2.23$, cod 90.62.5), and sputum eosinophil count ( $€ 27.17$, cod 91.39 .2 ). Each loss of a working day due to the disease was valued using the cost of paid and unpaid (household activities, caring for family members and others, and volunteering) work, specific for age and sex [20], updated to 2019 [21] and reported in Table S2 in Additional file 1. For non-workers (students, retired or unemployed workers), only the total length of hospital stay was multiplied by the cost of unpaid work.

\section{Statistical Analysis}

All resources (drug consumption, number of exacerbations, hospitalizations, unscheduled visits, diagnostic exams, and lost working days) were standardized to 12 months, divided the number of events by the person-years (PY) of follow-up pre-OMA, pre-MEP, and post-MEP.

Categorical variables are expressed as counts and percentages, continuous variables are summarized using mean and standard deviation (SD), and events (exacerbations with or without hospitalization) are expressed as absolute numbers and annual rates. Differences between pre- and post-MEP treatments were tested using generalized estimating equation (GEE) models [22], with a covariate of treatment period; for dichotomous outcomes and count data, we assumed Binomial and Poisson distributions, respectively. Specific relative effect measures were also presented with $95 \%$ confidence intervals (Cl): odds ratio (OR) for categorical outcomes, rate ratio (RR) for rates, and absolute difference for continuous outcomes, respectively. All statistical analyses were performed using STATA (StataCorp. 2017, release 15).

\section{Results}

A total of 33 patients, previously treated with OMA and switched to MEP, were enrolled in the study. Mean age was 56.8 years (SD $=11.3)$, and the majority of participants were women (84.8\%) with a familiar history of asthma (78.8\%); mean duration of asthma was 23.7 years (SD $=12.2)$, all patients were sensitized to perennial allergens, and $69.7 \%$ were sensitized to other allergens. All baseline characteristics are detailed in Table 1.

Page $3 / 9$ 
Table 1

Summary of demographic and baseline clinical characteristics of the enrolled cohort (continuous data are presented as mean \pm standard deviation, categorical data as absolute number and percentage in brackets).

\begin{tabular}{|ll|}
\multicolumn{2}{|c|}{ percentage in brackets). } \\
\hline Characteristics & $\mathbf{N}=33$ \\
\hline Age (years) & $56.8 \pm 11.3$ \\
\hline Gender (female) & $28(84.8 \%)$ \\
\hline Weight $(\mathrm{kg})$ & $69.5 \pm 12.9$ \\
\hline Body mass index $(\mathrm{kg} / \mathrm{m} 2)$ & $27.6 \pm 4.5$ \\
\hline Former smoker & $14(42.4 \%)$ \\
\hline Familiar history of asthma & $26(78.8 \%)$ \\
\hline Duration of asthma (years) & $23.7 \pm 12.2$ \\
\hline Atopy & $33(100 \%)$ \\
\hline Sensitized to perennial allergens & $33(100 \%)$ \\
\hline Sensitized to other allergens & $23(69.7 \%)$ \\
\hline Allergy comorbidities & \\
\hline Gastroesophageal reflux disease & $18(54.5 \%)$ \\
\hline Sensitized to acetylsalicylic acid & $6(18.2 \%)$ \\
\hline Nasal polyps & $20(60.6 \%)$ \\
\hline Allergic rhinitis & $13(39.4 \%)$ \\
\hline Bronchiectasis & $6(18.2 \%)$ \\
\hline Obstructive sleep apnea syndrome & $3(9.1 \%)$ \\
\hline
\end{tabular}

There was no apparent difference between pre-OMA and pre-MEP periods with regard to almost all clinical parameters (Table 2 and Fig. 1), with the exception of slightly lower numbers referring to annual exacerbation rate ( $\mathrm{RR}=0.76,95 \% \mathrm{Cl} 0.69$ to 0.85 ), hospitalizations ( $\mathrm{RR}=0.42,95 \% \mathrm{Cl} 0.22$ to 0.78 ) and total number of lost working days due to disease ( $\Delta=-3.4,95 \% \mathrm{Cl}-5.0$ to -1.8$)$.

Table 2

Clinical evolution of disease in the three periods considered in the analysis. Continuous data are reported as mean \pm standard deviation. Dichotomous data as absolute number (percentages), and relative measure as mean (95\% confidence interval).

\begin{tabular}{|c|c|c|c|c|c|}
\hline Parameter & Pre OMA & Pre MEP & Post MEP & Pre MEP vs pre OMA & Post MEP vs pre MEP \\
\hline Patients OCS-dependent & $27(81.8 \%)$ & $29(87.9 \%)$ & $4(12.1 \%)$ & $\mathrm{OR}=1.61(0.84$ to 3.11$)$ & $\mathrm{OR}=0.02$ (0.005 to 0.08$)$ \\
\hline Patients with $A C T \geq 20$ & $0(0 \%)$ & $0(0 \%)$ & $30(90.9 \%)$ & - & $\mathrm{OR}=2.43(0.12$ to 48.88$)$ \\
\hline FEV1 (L) & $1.81 \pm 0.65$ & $1.77 \pm 0.63$ & $1.96 \pm 0.67$ & $\Delta=-0.02(-0.08$ to 0.05$)$ & $\Delta=0.16(0.09$ to 0.24$)$ \\
\hline FEV1 (\%) & $75.1 \pm 15.6$ & $75.5 \pm 17.8$ & $82.3 \pm 14.3$ & $\Delta=0.01(-0.02$ to 0.05$)$ & $\Delta=0.06(0.03$ to 0.08$)$ \\
\hline $\mathrm{FVC}(\mathrm{L})$ & $2.63 \pm 0.83$ & $2.60 \pm 0.83$ & $2.75 \pm 0.89$ & $\Delta=-0.02(-0.1$ to 0.07$)$ & $\Delta=0.13(0.04$ to 0.22$)$ \\
\hline FVC (\%) & $90.8 \pm 14.3$ & $88.1 \pm 17.4$ & $95.2 \pm 14.5$ & $\Delta=-0.02(-0.06$ to 0.01$)$ & $\Delta=0.06$ (0.00 to 0.12$)$ \\
\hline FEV1/FVC (\%) & $68.5 \pm 11.8$ & $67.9 \pm 10.7$ & $71.7 \pm 10.5$ & $\Delta=0.00(-0.03$ to 0.02$)$ & $\Delta=0.03$ (0.01 to 0.06$)$ \\
\hline $\operatorname{lgE}(\mathrm{Ul} / \mathrm{ml})$ & $334 \pm 249$ & $354 \pm 261$ & $285 \pm 229$ & $\Delta=14.5(-27.4$ to 56.4$)$ & $\Delta=-54.9(-95.2$ to -14.6$)$ \\
\hline FeNO (ppb) & $36.7 \pm 21.3$ & $44.7 \pm 24.1$ & $32.8 \pm 17.6$ & $\Delta=6.8(2.4$ to 11.3$)$ & $\Delta=-11.3(-19.6$ to -3.0$)$ \\
\hline Eosinophils serum (cell/mcl) & $499 \pm 203$ & $538 \pm 223$ & $71.6 \pm 87.4$ & $\Delta=31.6(-25.9$ to 89$)$ & $\Delta=-466(-542$ to -391$)$ \\
\hline
\end{tabular}

After almost one year of MEP therapy (mean 11.7 months, SD = 3.6), improvements in patient clinical conditions were highly evident in comparison to the period of treatment with OMA in regard to exacerbation rate ( $\mathrm{RR}=0.06,95 \% \mathrm{Cl} 0.03$ to 0.14$)$, chronic $\mathrm{OCS}$ use $(\mathrm{OR}=0.02,95 \% \mathrm{Cl} 0.002$ to 0.08$)$, and lost working days due to disease $(\Delta=-7.9,95 \% \mathrm{Cl}-11.2$ to -4.6$)$, respectively. No subject needed hospitalization (Table 2 and Fig. 1$)$. Daily OCS dose in OCSdependent patients remained constant during the study period (Fig. 1). Furthermore, all main respiratory parameters improved (Table 2). Indeed, serum IgE 
levels decreased from 354 to $285 \mathrm{UI} / \mathrm{mL}(\Delta=-54.9,95 \% \mathrm{Cl}-95.2$ to -14.6$)$, FeNO from 44.7 to $32.8 \mathrm{ppb}(\Delta=-11.3,95 \% \mathrm{Cl}-19.6$ to -3.0$)$, and blood eosinophil count from 538 to 71.6 cells $/ \mu \mathrm{L}(\Delta=-466,95 \% \mathrm{Cl}-542$ to -391$)$, respectively.

The 12-month total costs referring to the two biological therapies were comparable (Table 3 and Fig. 2). The cost increment for drug therapy ( $€$ 1,581, $95 \%$ $\mathrm{Cl}-324$ to 3,486$)$ was due only to the cost of OMA (+18.8\%), while the cost of the other drugs slightly decreased $(-6.1 \%)$. The impact of switching to a newer biologic treatment was almost completely offset by $100 \%$ saving in exacerbation management ( $€-411,95 \% \mathrm{Cl}-727$ to 94$), 29.1 \%$ savings in visits/exams cost ( $€-198,95 \% \mathrm{Cl}-289$ to -107$)$, and $76.4 \%$ saving in productivity loss due to work absenteeism ( $€-572,95 \% \mathrm{Cl}-858$ to -286$)$.

Table 3

Total pre- and post-MEP annual costs. Annual costs are reported as mean \pm standard deviation, and annual delta as mean (95\% confidence interval).

\begin{tabular}{|llll|}
\hline & Pre MEP $(\boldsymbol{\epsilon})$ & Post MEP $(\boldsymbol{\epsilon})$ & Post vs pre MEP ( $\boldsymbol{\epsilon})$ \\
\hline Total cost & $12,239 \pm 961$ & $12,639 \pm 88$ & $400(-1,588$ to 2,389$)$ \\
\hline Drug therapy & $10,398 \pm 912$ & $11,979 \pm 74$ & $1,581(-324$ to 3,486$)$ \\
\hline Exacerbations & $411 \pm 155$ & 0 & $-411(-727$ to -94$)$ \\
\hline Visits and exams & $681 \pm 32$ & $483 \pm 20$ & $-198(-289$ to -107$)$ \\
\hline Productivity loss & $749 \pm 173$ & $177 \pm 47$ & $-572(-858$ to -286$)$ \\
\hline MEP: mepolizumab; OMA: omalizumab & \\
\hline
\end{tabular}

\section{Discussion}

OMA has been for many years the only therapeutic choice for non-controlled severe allergic asthmatic patients, sensitized to perennial allergens. Nowadays, it is possible to switch OMA non-responder patients to other available biological treatments such as MEP, introduced in Italy since 2017 [13]. In our study we enrolled 33 patients with severe eosinophilic allergic asthma, non-controlled by OMA, who switched to MEP. After 1 year of MEP therapy, we observed evident clinical benefits including significant decreases in asthma exacerbations, hospitalizations, OcS intake and working absenteeism. The most likely cause of unsatisfactory therapeutic response to OMA was probably related to the initial, obligatory choice of omalizumab due to unavailability of alternative biologics. In fact, at the beginning of this study and for more than the previous decade, OMA was the only available biologic therapy indicated for severe uncontrolled allergic asthmatics, and this drug was prescribed also in patients who did not represent the ideal targets [5]. Instead of OMA, some of these patients could likely better benefit from MEP as first-line biologic treatment. However, such alternative therapeutic options should not be considered as comparisons between anti-IgE and anti-IL-5 pharmacologic approaches. Rather, in consideration of the increasing availability of biologic therapies, it is very important to gain scientific evidence supporting the rationale underlying the most effective drug choice for each specific patient.

The efficacy of MEP in patients not optimally controlled by OMA was previously evaluated in the OSMO trial [11], and also in an Italian observational study [10]. Our results confirm the findings reported by both these studies. Moreover, we found significant decreases in exacerbation rate, hospitalization number, OCS-intake, and blood eosinophil count, which were very similar to those observed by Bagnasco, A few economic analyses regarding MEP have been published. A recent systematic literature review, aimed to investigate the cost-effectiveness of biological asthma treatments [3], identified just one paper evaluating the long-term clinical and economic impact of adding MEP to standard treatments with ICS and other controller medications on severe eosinophilic asthmatic US patients [5]. Despite the significant improvement in quality of life, the estimated cost-effectiveness of MEP exceeded value thresholds. Similar results were detected by the authors of a more recent study [23], but they conducted a further sensitivity analysis comparing MEP with OMA; in this "active" comparison, MEP resulted dominant (more effective and less costly) with respect to OMA. The annual cost of MEP, viewed from the US payer perspective, was also estimated on the basis of data published in MENSA trial [24]. The 12-month economic impact, not considering the cost of MEP, amounted to $\$ 1,277$ per patient, and hospitalization cost was the main expense. The difference with our findings was probably due to the different cost structure between US and Italy. Moreover, in the US study, indirect costs have not been included. The potential economic benefit provided by MEP could be underestimated because of two study limitations emerging from our retrospective investigation. First, OCS use has important delayed clinical consequences [2,25], and morbidity costs in OCS-dependent patients have a relevant impact on NHS [26,27]. Thus, a 12-month follow-up could not allow to really evaluate the potential savings due to OCS-intake reduction. Second, the number of lost working days because of severe asthma was reported only for employed subjects, whereas for unemployed patients we considered only the length of hospital stay (if a hospitalization occurred during the study). Therefore, working day losses were not recorded in non-hospitalized unemployed patients, even if productivity impairments were unavoidable in case of frequent exacerbations. Since female prevalence was greater than that observed in real-life [28], gender distribution was highly unbalanced in our study, and it is well known that the employment rate is lower for women in comparison to men. Hence, it is very likely that a more balanced population could have allowed to verify greater savings with regard to the cost due to productivity loss.

To the best of our knowledge this is the first study investigating not only the clinical benefits, but also the economic impact of switching to MEP patients not optimally controlled by OMA. The economic analysis included both direct health care costs (drug consumption, hospitalizations, diagnostic exams, unscheduled visits) and indirect costs (absenteeism due to disease), that represented a significant portion of the total burden of asthma [29,30]. According to our findings, productivity loss accounted for about $22 \%$ of the annual cost due to uncontrolled asthma (excluding OMA cost); such percentage decreased to $9 \%$ after one year of treatment with MEP (excluding MEP cost). 


\section{Conclusions}

In conclusion, our study provides evidence that patients with severe asthma, eligible for both OMA and MEP and not optimally controlled with the first of these two drugs, could improve their asthma control by switching to MEP. The economic impact due to such a therapeutic change is almost negligible for $\mathrm{NHS}$, because the cost increment due to drug price can be partially compensated by savings arising from decreases in exacerbation frequency, hospitalization rate, OCS use, and work absenteeism.

\section{Abbreviations}

\section{ACT}

asthma control test; Cl:confidence interval; DRG:Diagnosis Related Group; FENO:fractional exhaled nitric oxide; GEE:generalized estimating equation; GINA:Global Initiative for Asthma; HRQoL:health-related quality of life; ICS:inhaled corticosteroids; LABA:long-acting $\beta_{2}$-adrenergic agonists; MEP:mepolizumab; NHS:National Health System; OCS:oral corticosteroids; OMA:omalizumab; OR:odds ratio; PY:person-years; QoL:quality of life; RR:rate ratio; SABA:short-acting $\beta_{2}$-adrenergic agonists; SD:standard deviation

\section{Declarations}

\section{Ethics approval and consent to participate}

Ethical Committee approval was not needed because the drugs were prescribed according to current medical practice as part of the standard of care. All patients signed a written informed consent for the treatment of personal data (including scientific publications), which were anonymized.

\section{Consent for publication}

Not applicable

\section{Availability of data and materials}

The datasets used and/or analysed during the current study are available from the corresponding author on reasonable request.

\section{Competing interests}

Massimiliano Povero is employee of AdRes, which has received unconditional project funding from GSK. None for other authors.

\section{Funding}

Massimiliano Povero is employee of AdRes, which has received unconditional project funding from GSK.

\section{Author's contributions}

Giovanna Elisiana Carpagnano and Emanuela Resta equally contributed to the study conception and design. Material preparation were performed by Massimiliano Povero. Data collection were performed by Giovanna Elisiana Carpagnano, Corrado Pelaia, Mariella D’Amato, Nunzio Crimi, Nicola Scichilone, Giulia Scioscia, Onofrio Resta, Cecilia Calabrese, Girolamo Pelaia, and Maria Pia Foschino. The analysis were performed by Massimiliano Povero. The first draft of the manuscript was written by Giovanna Elisiana Carpagnano and Massimiliano Povero. All authors commented on previous versions of the manuscript and contributed to the final version. All authors read and approved the final manuscript.

\section{Acknowledgements}

Not applicable

\section{References}

1. Humbert M, Beasley R, Ayres J, et al. Benefits of omalizumab as add-on therapy in patients with severe persistent asthma who are inadequately controlled despite best available therapy (GINA 2002 step 4 treatment): INNOVATE. Allergy. 2005;60(3):309-16.

2. Canonica GW, Colombo GL, Bruno GM, et al. Shadow cost of oral corticosteroids-related adverse events: A pharmacoeconomic evaluation applied to real-life data from the Severe Asthma Network in Italy (SANI) registry. World Allergy Organization Journal Volume 12, Issue 1, 2019,100007 https://doi.org/10.1016/j.waojou.2018.12.001

3. McQueen RB, Sheehan DN, Whittington MD, et al.Cost-Effectiveness of Biological Asthma Treatments: A Systematic Review and Recommendations for Future Economic Evaluations. PharmacoEconomics volume 36, pages 957-971(2018) https://doi.org/10.1007/s40273-018-0658-x

4. Campbell JD, Spackman DE, Sullivan SD. The costs and consequences of omalizumab in uncontrolled asthma from a USA payer perspective. Allergy. 2010;65:1141e1148.

5. Whittington MD, McQueen RB, Ollendrof DA, et al. Assessing the value of mepolizumab for severe eosinophilic asthma: a cost-effectiveness analysis. Ann Allergy Asthma Immunol (2017) Vol 118, Issue 2, 220-225 http://dx.doi.org/10.1016/j.anai.2016.10.028 
6. Pelaia C, Calabrese C, Terracciano R, et al. Omalizumab, the first available antibody for biological treatment of severe asthma: more than a decade of real-life effectiveness. The Adv Respir Dis 2018 Jan-Dec; 12:1753466618810192

7. Ortega HG, Liu MC, Pavord ID, Brusselle GG, FitzGerald JM, Chetta A, et al. Mepolizumab Treatment in Patients with Severe Eosinophilic Asthma. N Engl J Med. 2014 Sep 25 [cited 2020 Jan 7];371(13):1198-207.

8. Pavord ID, Korn S, Howarth P, Bleecker ER, Buhl R, Keene ON, et al. Mepolizumab for severe eosinophilic asthma (DREAM): A multicentre, double-blind, placebo-controlled trial. Lancet. 2012;380(9842):651-9.

9. Menzella F, Galeone C, Bertolini F, et al. Innovative treatments for severe refractory asthma: how to choose the right option for the right patient? J Asthma Allergy. 2017 Aug 30;10:237-247. doi: 10.2147/JAA.S144100

10. Bagnasco DE, Manzella F, Caminati M, et al. Efficacy of mepolizumab in patients with previous omalizumab treatment failure: Real-life observation. Allergy. 2019 Dec;74(12):2539-2541. doi: 10.1111/all.13937

11. Chapman KR, Albers FC, Chipps B, et al. The clinical benefit of mepolizumab replacing omalizumab in uncontrolled severe eosinophilic asthma. Allergy. 2019;74:1716-1726. doi: 10.1111/all.13850

12. Global strategy for asthma management and prevention (2019 update) https://ginasthma.org/wp-content/uploads/2019/06/GINA-2019-main-reportJune-2019-wms.pdf Accessed April 2020

13. Summary of Product Characteristics (SPC) mepolizumab [Internet]. Available from: https://www.ema.europa.eu/en/documents/productinformation/nucala-epar-product-information_en.pdf Accessed April 2020

14. Summary of Product Characteristics (SPC) omalizumab [Internet]. Available from: https://www.ema.europa.eu/en/documents/productinformation/xolair-epar-product-information_en.pdf Accessed April 2020

15. Informatore farmaceutico 2020 [Internet]. Available from: codifa.it Accessed April 2020

16. Anagrafica SORESA [Internet]. Available from: https://www.soresa.it/imprese/Pagine/Anagrafica-Beni.aspx Accessed April 2020

17. Lista farmaci equivalenti [Internet]. Available from: http://www.agenziafarmaco.gov.it/content/liste-di-trasparenza-e-rimborsabilità Accessed April 2020

18. Bollettino Ufficiale della Regione (BUR) Campania - Deliberazione giunta regionale n 270 del 12.06 .2012 [Internet]. Available from: http://burc.regione.campania.it/eBurcWeb/BurcPdfOutput/Burc_2012_6_26_11_15.pdf;jsessionid=FBD71BD1D08E8E2D8A98142BA07C4740.burcfe2 Accessed April 2020

19. GU Serie Generale n.23 del 28-01-2013 - Suppl. Ordinario n. 8. Decreto 18 ottobre 2012. Remunerazione prestazioni di assistenza ospedaliera per acuti, assistenza ospedaliera di riabilitazione e di lungodegenza post acuzie e di assistenza specialistica ambulatoriale. (13A00528) [Internet]. Available from https://www.gazzettaufficiale.it/eli/id/2013/01/28/13A00528/sg Accessed April 2020

20. Pradelli L, Ghetti G. A general model for the estimation of societal costs of lost production and informal care in Italy. Farmeconomia Heal Econ Ther pathways. 2017 Feb 21;18(1)

21. Harmonized Indices of Consumer Prices (HICP), European Commission EuroStat 2020 [Internet]. Available from: https://ec.europa.eu/eurostat/web/hicp/data/main-tables Accessed April 2020

22. Keene ON, Davis RL, Koch GG. Use of generalized estimating equations in a trial in influenza to explore treatment effects over time. Pharm Stat. 2004;3(4):281-287

23. Maunoury F, Pribil C, Aubier M, et al. Cost-effectiveness of mepolizumab in severe eosinophilic asthma patients in France. Value in Health volume 21, supplement 3, s413, october 01, 2018. https://doi.org/10.1016/j.jval.2018.09.2449

24. Basu A, Dalal A, Canonica GW, et al. Economic analysis of the phase III MENSA study evaluating mepolizumab for severe asthma with eosinophilic phenotype. Expert Rev Pharmacoecon Outcomes Res. 2017 Apr;17(2):121-131 doi: 10.1080/14737167.2017.1298444

25. Heffler E, Bagnasco D, Canonica GW. Strategies to reduce corticosteroid-related adverse events in asthma. Curr Opin Allergy Clin Immunol. 2019;19(1):61-7.

26. Heffler E, Blasi F, Latorre M, Menzella F, Paggiaro P, Pelaia G, et al. The Severe Asthma Network in Italy: Findings and Perspectives. J Allergy Clin Immunol Pract. 2019 May 1;7(5):1462-8.

27. Senna G, Guerriero M, Paggiaro PL, Blasi F, Caminati M, Heffler E, et al. SANI-Severe Asthma Network in Italy: A way forward to monitor severe asthma. Clin Mol Allergy. 2017 Apr 10;15(1).

28. Bagnasco D, Milanese M, Rolla G, Lombardi C, Bucca C, Heffler E, et al. The North-Western Italian experience with anti IL-5 therapy amd comparison with regulatory trials. World Allergy Organ J. 2018;11(1).

29. Cisternas MG, Blanc PD, Yen IH, et al. A comprehensive study of the direct and indirect costs of adult asthma. J Allergy Clin Immunol. 2003 Jun;111(6):1212-8

30. Souliotis K, Kousoulakou H, Hillas G, et al. Direct and indirect costs of asthma Management in greece: an expert Panel approach. Front. Public Health 5:67. doi: 10.3389/fpubh.2017.00067

\section{Figures}



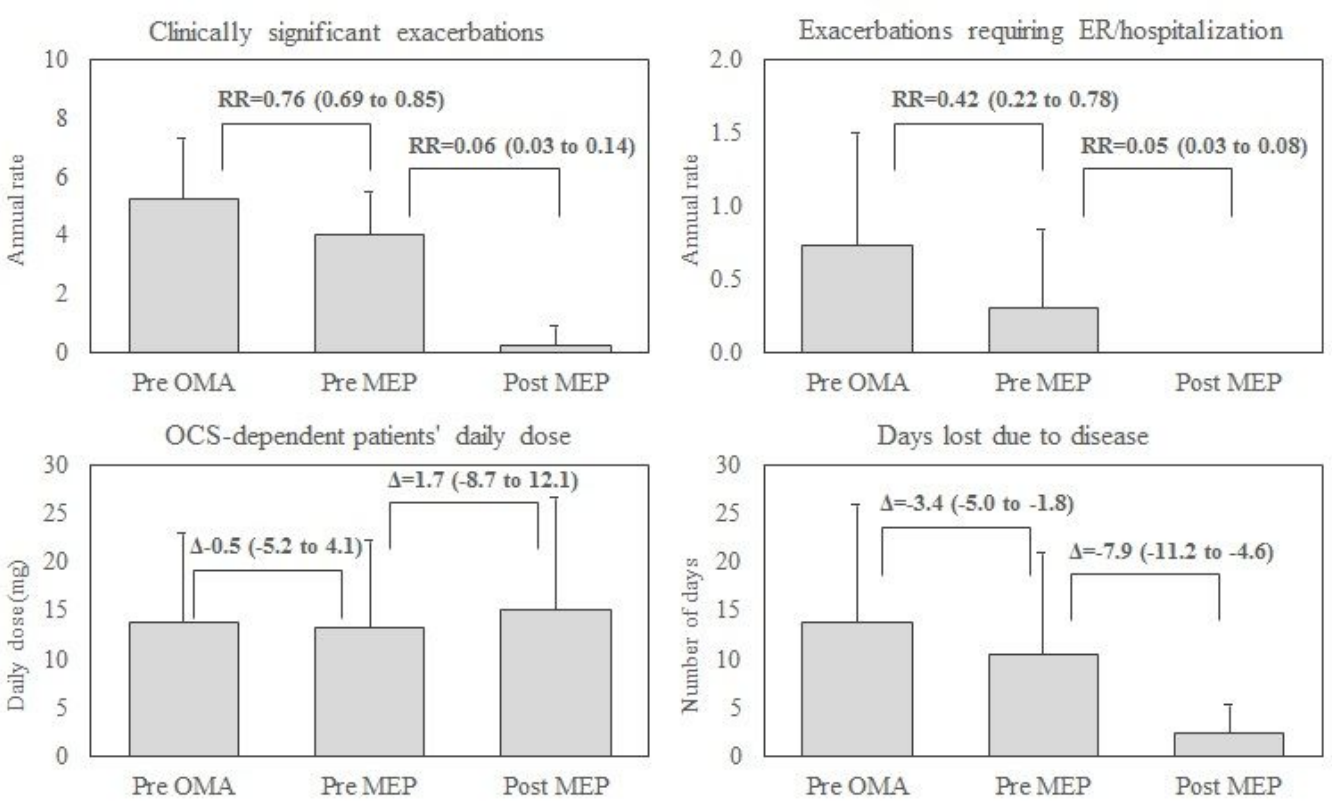

Figure 1
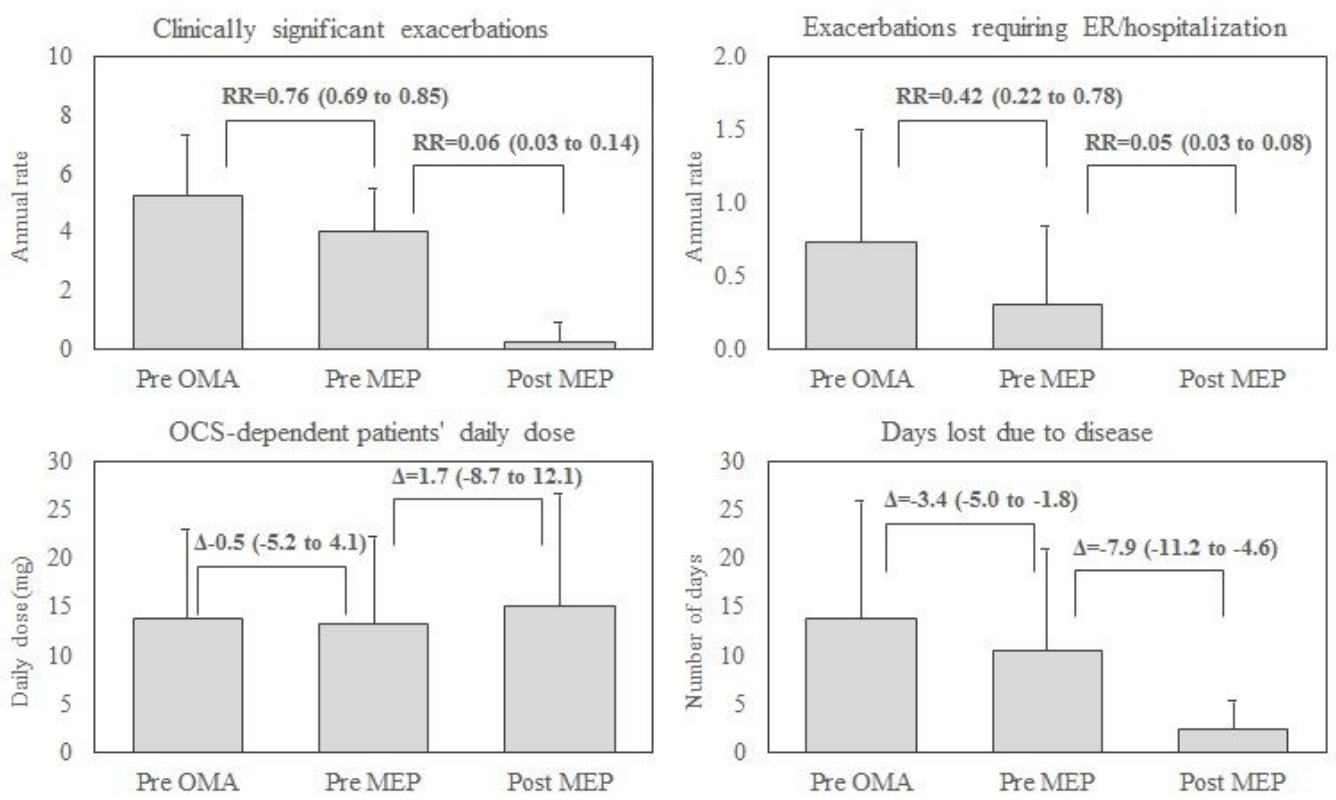

Figure 1

Annual impact post vs pre MEP

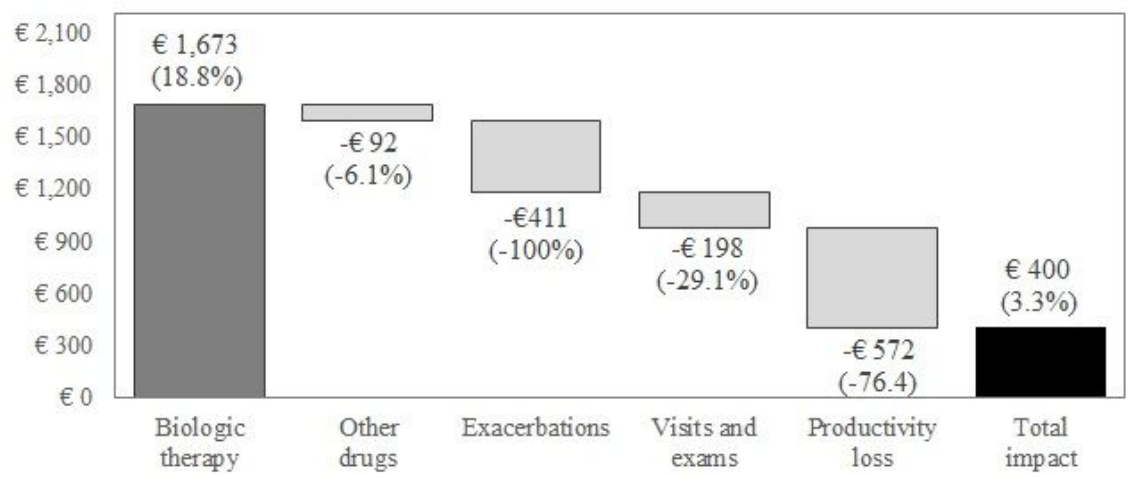

Figure 2 
Annual impact post vs pre MEP

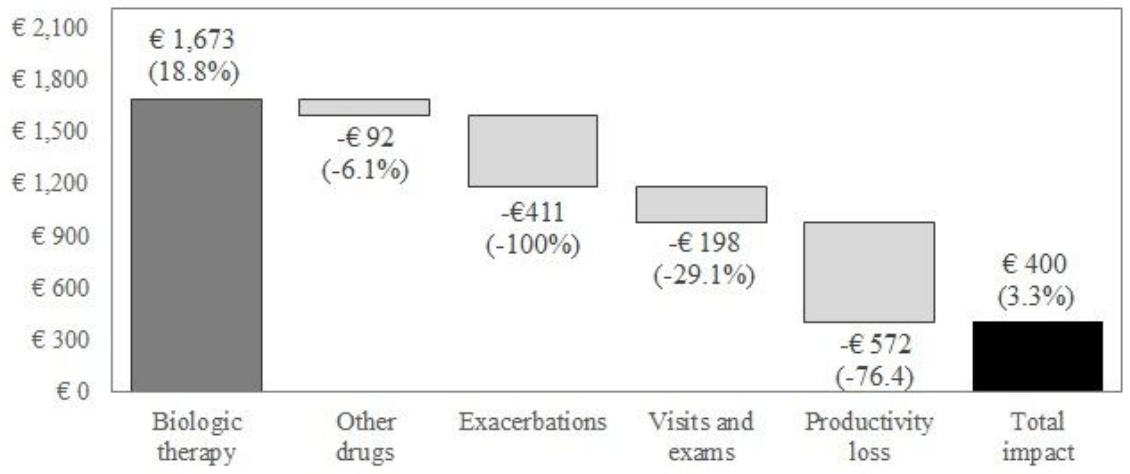

Figure 2

\section{Supplementary Files}

This is a list of supplementary files associated with this preprint. Click to download.

- Additionalfile1.docx

- Additionalfile1.docx 